\title{
Research and Review on Modified Novel SC-FDE Systems through Analyzing Synchronization and Parameter Estimation
}

\author{
Wenzhun Huang ${ }^{1, a^{*}}$, Yuting Zhang ${ }^{1}$, Xinxin $X^{1}{ }^{1}$ and Shanwen Zhang ${ }^{1}$ \\ ${ }^{1}$ Department of Electronic Information Engineering, Xijing University, Xi'an 710123, China \\ ahuangwenzhun@xijing.edu.cn, b912482316@qq.com, ${ }^{\text {c3} 346148500 @ q q . c o m, ~ d w j d w 716 @ 163 . c o m ~}$
}

Keywords: Communication Techniques, SC-FDE Systems, Parameter Estimation, Synchronization Scheme, Simple Weighted Least Square.

\begin{abstract}
With the bursting development of electricity communication and information technology, SC-FDE related techniques have been popularly applied to many modern wireless communication systems. This article is in view of the SC-FDE technique in the application of multipath time-varying airmail channel, an improved method of coarse timing synchronization. Traditional SC-FDE communication method, the channel estimation without using the underwater acoustic channel is one of the characteristics of sparse channel, channel estimation precision is low, the modified SC-FDE can overcome the shortcomings of OFDM system, and while maintaining the same complexity, to approximate the performance of OFDM system. Selection of the training symbol structure and the selection of training sequences directly affect the accuracy of coarse timing synchronization, finally affects the overall performance of SC-FDE system. The experimental result proves the effectiveness and robustness of the proposed method. The future work and prospect is also discussed.
\end{abstract}

\section{Introduction}

In the wireless digital communication system, to overcome the multipath fading signals there are some meaningful techniques such as: Orthogonal Frequency Division Multiplexing (OFDM) and Single Carrier-Frequency Domain Equalization (SC-FDE). OFDM has high spectrum efficiency, strong ability to resist multipath fading and balance the advantages of simple, and have been widely used in wired and wireless communications, but sensitive to carrier synchronization, power than high peak [1]. SC-FDE can overcome the shortcomings of OFDM system, and while maintaining the same complexity, to approximate the performance of OFDM system. Therefore, research on OFDM with SC-FDE application in various environments and comparing the performance between them become a hotspot in recent years. Coarse timing synchronization is the beginning of the SCFDE system timing synchronization, by adopting appropriate capture algorithm and circuit, determine the receiver fast Fourier transform to the starting position of the window [2]. So, to ensure that the system based on SC - FDE aviation communication good performance, fast coarse timing synchronization algorithm is of vital importance. The coarse timing synchronization algorithms usually use special structure of the training symbol repetition characteristics, by searching the timing roughly measure peak FFT the starting position of the window. Selection of the training symbol structure and the selection of training sequences directly affect the accuracy of coarse timing synchronization, finally affects the overall performance of SC-FDE system [3]. The SWLS method has been proven to be more robust to multipath channels than LLS method, because it weights the received signal with estimated power of channel frequency response such that the estimation error due to deep-fading frequency response can be minimized [4]. Traditional SC-FDE communication method, the channel estimation without using the underwater acoustic channel is one of the characteristics of sparse channel, channel estimation precision is low. Sparse channel refers to the time domain impulse response of channel with long time delay and contains a large deal of zeros [5].

In this paper, we conduct theoretical research based on the cyclic prefix of SC-FDE signal parameter estimation problem, a cyclic autocorrelation based method is proposed and its cyclic prefix length and the length of the useful signal blind estimation, and the known symbol rate and the number 
of useful signal symbol of conditions, the estimated number of cyclic prefix symbol. The experiment result shows the effectiveness of the proposed framework. The detailed is discussed below.

\section{The Proposed System and Technique}

The System Model. SC-FDE timing synchronization includes when partial capture and tracking. When partial capture which is also called coarse synchronization could be considered by the capturing of the appropriate algorithm and the original position of the circuit, we could determine the sampling data frame. Coarse timing synchronization is the early stage of the SC - FDE timing synchronization is mainly used in partial quantity is relatively large when the initial process of communication. At this time to guarantee the normal work of the communication system must be timely, quickly and accurately estimate the time bias and to compensate. Algorithm based on the special training symbol utilization rate of the frequency band of UW without inserting algorithm is high, but its ability to resist multipath interference is strong, and can rapidly estimate the timing error. Coarse timing synchronization requirements quickly and accurately, at the same time, more serious air channel multipath interference, considering these two points, this article in aeronautical communication system based on SC-FDE choose coarse timing synchronization algorithm based on the special training symbols. SC-FDE system based on the special training symbol frame structures are usually put training symbol before transmitting data, used to produce the timing synchronization information you need. The structure of the training symbol value choice and symbol has a decisive role on the whole performance of the algorithm. Therefore, coarse timing synchronization in SC-FDE plays a key role. When partial track denoted as the fine synchronization is taken into consideration by tracking algorithm, we could furtherly reduce the time deviation. The system is shown in the Fig. 1.

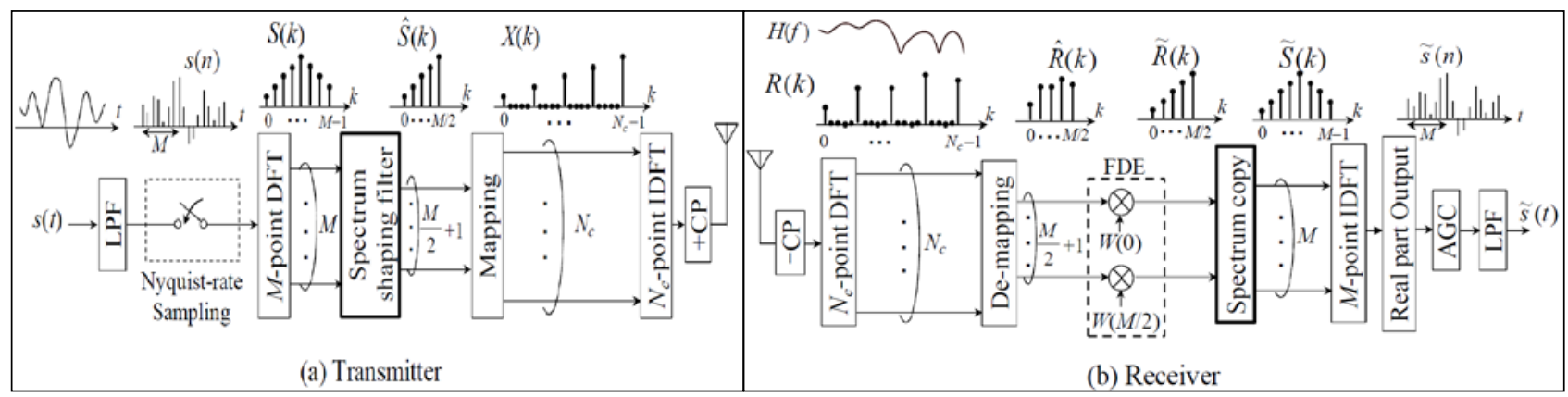

Fig. 1 The System Organization Description

The Transmit Signal Representation. The frequency components (referred to as subcarriers) in USB, which have the complex conjugate relation with that in LSB is shown in [2] which are removed. Due to the cyclic autocorrelation estimate signal data is limited, signal observation directly affect the estimation precision of cyclic autocorrelation length, at the same time also to the estimation precision of the algorithm in this paper. Therefore, the output includes only the subcarriers in LSB which are expressed as the formula $1 \sim 2$. When making related, the existence of the $\mathrm{CP}$ can make timing measure peak platform. Under the condition of no noise and distortion of the ideal channel, peak platform more smooth and clear, the impact on the timing accuracy is not big.

$$
\begin{aligned}
& \hat{S}(k)=\sqrt{Q} \times \frac{1}{\sqrt{M}} \sum_{n=0}^{M-1} s(n) \exp \left(-j 2 \pi \frac{n}{M}\right) \\
& x(n)=\frac{1}{\sqrt{N_{c}}} \sum_{k=0}^{N_{c}-1} X(k) \exp \left(j 2 \pi \frac{k}{N_{c}}\right)
\end{aligned}
$$

But air channel of multipath and Doppler frequency shift phenomenon, the algorithm of timing measures peak will decline and the starting position of the peak platform will appear fuzzy phenomenon, can't get accurate timing synchronization information. In order to gain accurate timing of synchronization information, need to reduce the timing measurement platform fuzzy phenomenon, improve the timing measure peak is sharp. 
The Proposed Model and Systematic Description. Each source contains the general minimum recommended forward current according to the data sheet, it is current and optical emission. Due to the hot side, AC/pulse current must be according to manufacturer's limited data manual, to ensure that the chip does not overheat, in order to avoid the E/O efficiency degradation or, in the worst case, a total failure. Through on the frequency of the sequence is even send a pseudo random data, send zero data on the sequence for the frequency of the odd number, and then by IFFT transformation, can get the required training symbol. The training symbol in the frequency domain to send vector can be simply represented as:

$$
C=\left[c_{0}, 0, c_{2}, 0, \ldots, c_{2 i}, 0, \ldots c_{N-2}, 0\right]
$$

Algorithm training symbol can be divided into equal length of two parts, the two parts corresponding to the value of the identical. Using the symmetry of the training symbol before and after two parts for sliding correlation of received signal, timing is obtained by search related peak point estimation deviation. Symbol timing measurement function is defined as:

$$
\begin{aligned}
& P(d)=\sum_{m=0}^{N / 2-1} r(d+N / 2+m) \times r^{*} \times(d+m) \\
& R(d)=\sum_{m=0}^{N / 2-1}|r(d+N / 2+m)|^{2}
\end{aligned}
$$

The structure of the training sequence is selected, training sequences CAZAC sequence. CAZAC sequence has two obvious features: sequence in time domain and frequency domain are constant envelope, make its ability to resist noise is very strong; The sequence of the autocorrelation function at other than zero point is very small, has the good phase characteristic. Excellent characteristics of CAZAC sequence to improve the performance of SC-FDE system coarse timing synchronization algorithm and its mathematical description is as follows:

$$
\mu(n)=\frac{1}{k} \frac{\sqrt{2 P Q}}{M}\left\{\sum_{k=0}^{M / 2} H(k)\left[\sum_{n^{\prime}=0}^{M-1} s\left(n^{\prime}\right) \exp (j 2 \pi)\left(n-n^{\prime}\right) / M\right]\right\}
$$

Algorithm in two scenarios of air channel is a big platform for the peak and the starting position of the peak platform is very fuzzy, difficult to determine the exact timing. And the peak in the improved algorithm eliminated platform phenomenon, timing measure peak is very sharp. A new algorithm to overcome the influence of air channel multipath interference, regular measure under various channel scene model there is a sharp peak, to make more accurate timing. Under channel and error-free reference feedback signal becomes the formula 6 . This method use the matching tracking idea, can accurately estimate the time domain impulse response of the underwater acoustic channel with random number as the pilot, to avoid the problem that traditional methods must be special pilot. Insert the UW algorithm using UW instead of cyclic prefix, the use of the characteristics of UW channel estimation and equalization which raises the utilization ratio of the frequency band. Insert UW algorithm of defect is need a long time to estimate the timing deviation, secondly when interference between two frames, timing is not accurate.

$$
M_{i}[k] \approx \frac{\exp (j 2 \pi(N-1)(\varepsilon+k \delta) / N)}{N}\left(\frac{\eta_{i}}{1+\eta_{i}}\right)\left|X_{i}[k]^{2}\right|
$$

\section{Experimental Analysis and Simulation}

This section we simulate the channel scenario in aviation model, for the proposed SC-FDE system based coarse timing synchronization methods are simulated, and compared with algorithm performance comparison. We depict RMSEs of joint RCFO and STO estimations under different levels of RCFOs where STO and SNR are fixed at 20ppm and $24 \mathrm{~dB}$, respectively. With the property of the SC - FDE signal cycle smoothly on the signal parameter estimation problem is studied and realized with SC-FDE blind estimation of signal parameters. Compared with the existing algorithms, 
this algorithm improves the estimation accuracy. Has no cycle stability due to noise, this algorithm in low SNR when also can have a better estimation performance. The result is shown in the Fig. 2.
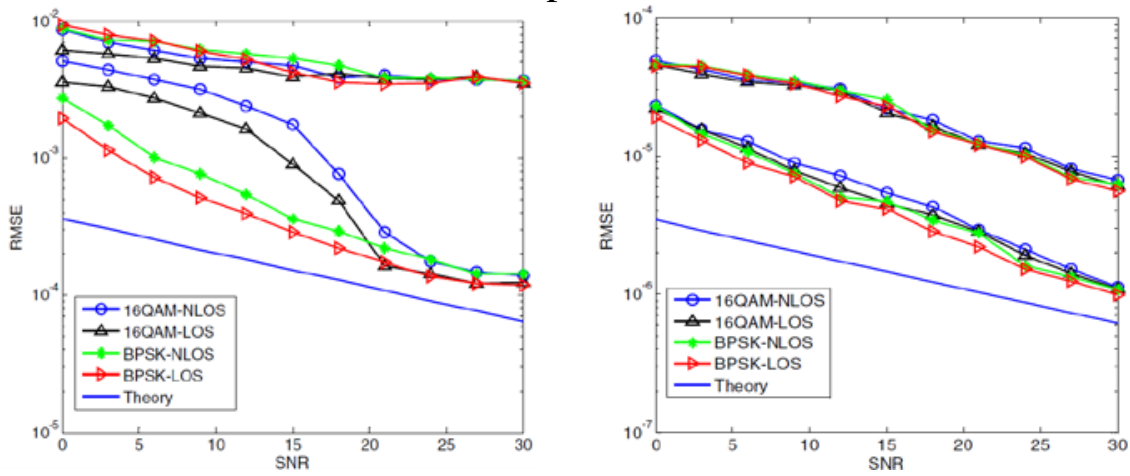

Fig. 2 The Experimantal Result for the System

\section{Summary and Conclusion}

This article is in view of the SC-FDE technique in the application of multipath time-varying airmail channel, an improved method of coarse timing synchronization. Good performance through the use of CAZAC sequence and choose suitable training symbol structure, eliminate the phenomenon of the traditional algorithm of time measure peak platform. SC-FDE can overcome the shortcomings of OFDM system, and while maintaining the same complexity, to approximate the performance of OFDM system. Therefore, research on OFDM with SC-FDE application in various environments and comparing the performance between them become a hotspot in recent years. The experiment result proves the effectiveness of our proposed methodology and in the future we plan to conduct more related literature review to modify our current approach.

\section{Acknowledgements}

This work is financially supported by the scientific research program of Shaanxi Provincial Education Department (Program No. 14JK2156).

\section{References}

[1] Elgala, Hany, and Thomas DC Little, Polar-Based OFDM and SC-FDE Links Toward Energy-Efficient Gbps Transmission Under IM-DD Optical System Constraints, Journal of Optical Communications and Networking 7, no. 2 (2015) A277-A284.

[2] Chi, Nan, Yuanquan Wang, Yiguang Wang, Xingxing Huang, and Xiaoyuan Lu, Ultra-high-speed single red-green-blue light-emitting diode-based visible light communication system utilizing advanced modulation formats. Chinese Optics Letters 12, no. 1 (2014) 010605.

[3] Ribeiro, Filipe Casal, Rui Dinis, Francisco Cercas, and Adão Silva, Receiver design for the uplink of base station cooperation systems employing SC-FDE modulations, EURASIP Journal on Wireless Communications and Networking 2015, no. 1 (2015) 1-15.

[4] Jin, Yongnu, Daehan Kwak, Kyeong Jin Kim, and Kyung Sup Kwak, Cyclic Prefixed Single Carrier Transmission in Intra-Vehicle Wireless Sensor Networked Control Systems, In Vehicular Technology Conference (VTC Spring), 2014 IEEE 79th, pp. 1-5.

[5] Gomes, Rodolfo, Zaid Al-Daher, Akram Hammoudeh, Khaled Sobaihi, Rafael Caldeirinha, and Telmo Fernandes, Performance and evaluation of OFDM and SC-FDE over an AWGN propagation channel under RF impairments using simulink at 60GHz, In Antennas and Propagation Conference (LAPC), 2014 Loughborough, pp. 685-689. 